\title{
Velocity Estimation Methods with Applications to Tracking Control of a Harmonic Drive System
}

\author{
Yu-Sheng Lu ${ }^{\mathrm{a},},{ }^{*}$, Jhong-Sian Lin ${ }^{\mathrm{b}}$

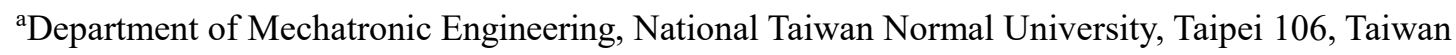 \\ ${ }^{b}$ Department of Mechanical Engineering, National Yunlin Univerity of Science \& Technology, Yunlin 640, Taiwan \\ *Corresponding Author: luys@ntnu.edu.tw
}

\begin{abstract}
In this paper, the effects of velocity estimation on the tracking performance of a harmonic drive system are investigated. Harmonic drive gearing is an important element to precision motion systems that are needed to have lightweight. However, the harmonic drive gearing introduces compliance to the motion systems, and velocity feedback is required to increase system damping. In this paper, several nonmodel-based approaches to velocity estimation are revisited and practically applied to the tracking control of a harmonic drive system. Based on experimental results, their performance comparisons are done in terms of tracking precision and smoothness of estimation results.
\end{abstract}

Keywords: velocity estimation, harmonic drive gearing.

\section{Introduction}

The quality of a velocity signal is crucial to motion control systems. Because velocity feedback can be used to increase relative stability of a closed-loop motion control system, leading to high damping and large bandwidth compared to those without velocity feedback. However, estimating a velocity in a motion control system precisely is as difficult as forecasting an instantaneous price trend in a stock market. Many studies have been conducted to devise various schemes of velocity estimation, which can be roughly divided into two category. One category is model-based approaches whereas the other is non-model-based approaches ${ }^{(1)}$. The model-based approaches require a dynamic model of the plant to be controlled. However, there always exist uncertainties associated with the plant's model, which deteriorates effectiveness of the model-based approaches. This paper focuses on applications of the nonmodel-based approaches that need no mathematical model of the plant.

Harmonic drive gearing is an important element of a precise motion system ${ }^{(2)}$. It features small size with light weight, high gear ratio and, most importantly, small backlash. Its compactness with virtually no backlash makes it perfect for lightweight motion systems, such as lightweight service robots. Nevertheless, the harmonic drive gearing introduces compliance to motion systems, increases flexibility of motion systems and reduces structural damping of motion systems. Thus, velocity feedback is rather important to motion systems with harmonic drive gearing in order to increase damping. Zhu implements a velocity estimation solution on an FPGA (field-programmable gate array) device for a harmonic drive system $^{(3)}$. Based on experimental results, it was concluded that the positional control accuracy of a harmonic drive is more sensitive to the phase lag than to the smoothness in velocity estimation $^{(3)}$. This paper investigates effectiveness of several non-model-based approaches to velocity estimation on positional control of a harmonic drive system.

Due to the flexibility introduced by a harmonic drive gearing, a harmonic drive system is modeled as a compliant-load system, which is also referred to as a two-mass system. The model of a compliant-load system consists of two subsystems, in which two subsystems are coupled by a spring. One subsystem model describes the actuator/motor side whereas the other describes the load side. For a harmonic drive system, Cai et al. proposed a control scheme with double speed loops, consisting of one speed loop for the motor side and the other for the load side ${ }^{(4,5)}$. Moreover, the speed loop for the motor side is an 
inner loop whereas the speed loop for the load side is an outer loop. A subsystem backstepping design (SSBD) that is an improved version of conventional integrator backstepping design (CIBD) has been proposed ${ }^{(6)}$. The SSBD is an appropriate approach to designing a feedback controller for a harmonic drive system and is adopted in this paper for investigating effectiveness of several non-model- based approaches to velocity estimation.

This paper is organized as follows. Section 2 describes non-model-based approaches to velocity estimation which are used in this paper. Section 3 provides a model of a harmonic drive system and its SSBD design. Section 4 describes an experimental harmonic drive system and reports experimental results. Finally, conclusions are given in Section 5.

\section{Non-Model-Based Velocity Estimation}

\subsection{Finite Difference Method (FDM)}

The FDM is also referred to as the fixed-time method. In the case of periodic sampling, the FDM constantly takes the first-order difference of position with respect to time as its velocity estimate. That is, the FDM produces a velocity estimate given by

$$
\hat{v}_{\mathrm{FD}}\left(n T_{s}\right)=\frac{x\left(n T_{s}\right)-x\left(n T_{s}-T_{s}\right)}{T_{s}},
$$

in which $T_{s}$ denotes the sampling period and $x\left(n T_{s}\right)$ is the measured position at $t=n T_{s}$. The FDM is simple and easy to implement. However, this method deteriorates in a low-speed situation.

\subsection{Inverse-Time Method (ITM)}

The ITM is also referred to as the fixed-distance method, which is usually used in applications where displacement is sensed by an incremental optical/magnetic encoder. The encoder produces a pulse every fixed displacement. The ITM uses a high-frequency clock to measure the time elapsed between two successive pulses, and the velocity estimate is obtained by dividing the fixed displacement by the measured time, in which the fixed displacement is also the positional resolution of the incremental encoder. That is, the ITM produces a velocity estimate given by

$$
\hat{v}_{\mathrm{IT}}(t)=\frac{\Delta_{x}}{T_{\Delta}}
$$

in which $\Delta_{x}$ is the sensor resolution that represents the fixed distance between two consecutive pulses, and $T_{\Delta}$ denotes the time between these two pulses. The ITM usually gives less phase lag than the FDM in velocity estimation. However, in a high-speed situation, $T_{\Delta}$ becomes small, and its measurement has more quantization error due to a finite frequency of the counting clock, leading to less signal-to-noise ratio.

\subsection{Tracking Differentiator (TD)}

The TD is an observer with a bang-bang correcting term, in which the bang-bang action is determined by a nonlinear switching function that is commonly used in a time-optimal control law. The TD law is described by ${ }^{(7)}$

$$
\begin{aligned}
& \dot{\hat{x}}_{\mathrm{TD}}=\hat{v}_{\mathrm{TD}}, \\
& \dot{\hat{v}}_{\mathrm{TD}}=-\alpha \operatorname{sgn}\left(\hat{x}_{\mathrm{TD}}-x+\frac{\hat{v}_{\mathrm{TD}}\left|\hat{v}_{\mathrm{TD}}\right|}{2 \alpha}\right),
\end{aligned}
$$

in which $\alpha$ is a design parameter, and $\hat{x}_{\mathrm{TD}}$ and $\hat{v}_{\mathrm{TD}}$ respectively denote the position and the velocity estimates of the TD.

\subsection{State-Space Velocity Observer (SSVO)}

The SSVO is a linear observer similar to the Luenburger observer ${ }^{(8)}$. However, the SSVO is augmented with an acceleration signal. The SSVO can be described by

$$
\begin{aligned}
& \dot{\hat{x}}_{\mathrm{SS}}=\hat{v}_{\mathrm{SS}}+L_{1}\left(x-\hat{x}_{\mathrm{SS}}\right), \\
& \dot{\hat{v}}_{\mathrm{SS}}=a+L_{2}\left(x-\hat{x}_{\mathrm{SS}}\right),
\end{aligned}
$$

in which $a$ denotes the measured acceleration, $L_{1}$ and

$L_{2}$ are constant observer gains, and $\hat{x}_{\mathrm{SS}}$ and $\hat{v}_{\mathrm{SS}}$ respectively denote the position and the velocity estimates of the SSVO. The characteristic polynomial of the SSVO is $s^{2}+L_{1} s+L_{2}$, which can be used to determine the observer gains, $L_{1}$ and $L_{2}$. 
The DCVO is also a linear observer but with a dynamic compensator, $C(s)^{(9,10)}$. The DCVO is

$$
\begin{aligned}
& \dot{\hat{x}}_{\mathrm{DC}}=\hat{v}_{\mathrm{DC}}, \\
& \dot{\hat{v}}_{\mathrm{DC}}=a+C *\left(x-\hat{x}_{\mathrm{DC}}\right),
\end{aligned}
$$

in which the ' $*$ ' denotes convolution, and $\hat{x}_{\mathrm{DC}}$ and $\hat{v}_{\mathrm{DC}}$ respectively denote the position and the velocity estimates of the DCVO. The dynamic compensator is designated as $C(s)=\left(c_{2} s+c_{1}\right) /\left(s+c_{3}\right)$, in which $c_{1}, \quad c_{2}$ and $c_{3}$ are constant design parameters $^{(9)}$. The characteristic polynomial of the DCVO is $s^{3}+c_{3} s^{2}+c_{2} s+c_{1}$, which can be used to determine the design parameters, $c_{1}, c_{2}$ and $c_{3}{ }^{(9)}$.

\section{Model of a Harmonic Drive System and its SSBD Design}

\subsection{Model of a Harmonic Drive System}

A harmonic drive gear comprises a wave generator, a flexible spline and a circular spline. The flexible spline introduces compliance between the motor side and the load side. Fig. 1 shows a schematic of the harmonic drive system, in which $J_{m}$ and $J_{l}$ are moment of inertia of the motor and the load, respectively; $D_{m}$ and $D_{l}$ are viscous coefficients of the motor and the load, respectively; $\theta_{m}$ and $\theta_{l}$ are angular positions of the motor and the load, respectively; $\tau_{f}$ and $\tau_{d}$ are disturbances at the motor and the load sides, respectively; $D_{h}$ and $K_{h}$ are viscous coefficient and spring constant, respectively, of the flexible spline; $\tau_{m}$ denotes the torque produced by the motor and is the control input to the plant; $m g l$ is due to gravitational effect. Moreover, $\tau_{l}$ is the joint torque that is

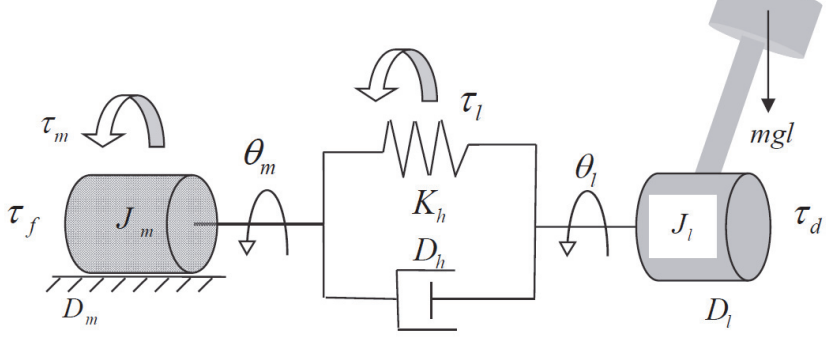

Fig. 1. Schematic of a harmonic drive system.

measurable in our experimental setup. Define a state variable as

$$
\tilde{\tau}_{l}=\frac{\tau_{l}}{J_{l}}+\frac{D_{h}}{J_{l} K_{h}} \dot{\tau}_{l}
$$

The model of the harmonic drive system is given by ${ }^{(6)}$

$$
\begin{aligned}
& \ddot{\theta}_{l}=\tilde{\tau}_{l}-\frac{\tau_{d}}{J_{l}}-\frac{D_{l}}{J_{l}} \dot{\theta}_{l}+\frac{m_{l} g l \sin \theta_{l}}{J_{l}}, \\
& \ddot{\widetilde{\tau}}_{l}=u+f+d_{2},
\end{aligned}
$$

in which

$$
u=\frac{D_{h}}{J_{m} J_{l}} \dot{\tau}_{m}+\left(\frac{K_{h}}{J_{m} J_{l}}-\frac{D_{m} D_{h}}{J_{m}^{2} J_{l}}-\frac{D_{h}^{2}}{J_{m} J_{l}}\left(\frac{1}{J_{m}}+\frac{1}{J_{l}}\right)\right) \tau_{m},
$$

$$
\begin{aligned}
& f=\left(\frac{D_{h} D_{m}^{2}}{J_{l} J_{m}^{2}}-\frac{K_{h} D_{m}}{J_{l} J_{m}}+\frac{D_{h}^{2} D_{m}}{J_{l} J_{m}}\left(\frac{1}{J_{m}}+\frac{1}{J_{l}}\right)\right) \dot{\theta}_{m} \\
& +\left(\frac{D_{h} D_{m}}{J_{m}^{2} J_{l}}-\frac{K_{h}}{J_{l}}\left(\frac{1}{J_{m}}+\frac{1}{J_{l}}\right)+\frac{D_{h}^{2}}{J_{l}}\left(\frac{1}{J_{m}}+\frac{1}{J_{l}}\right)^{2}\right) \tau_{l} \\
& +\left(\frac{D_{h}^{2} D_{m}}{J_{m}^{2} J_{l} K}-\frac{2 D_{h}}{J_{l}}\left(\frac{1}{J_{m}}+\frac{1}{J_{l}}\right)+\frac{D_{h}^{3}}{J_{l} K_{h}}\left(\frac{1}{J_{m}}+\frac{1}{J_{l}}\right)^{2}\right) \dot{\tau}_{l}
\end{aligned}
$$

$$
\begin{aligned}
& +\left(\frac{D_{h}^{2}}{J_{l}^{2}}\left(\frac{1}{J_{m}}+\frac{1}{J_{l}}\right)-\frac{K_{h}}{J_{l}^{2}}\right) m g l \sin \theta_{l}-\frac{D_{h}}{J_{l}^{2}} \dot{\theta}_{l} m g l \cos \theta_{l}, \\
& d_{2}=\frac{D_{h}}{J_{m} J_{l}} \dot{\tau}_{f}+\left(\frac{K_{h}}{J_{m} J_{l}}-\frac{D_{h} D_{m}}{J_{m}^{2} J_{l}}-\frac{D_{h}^{2}}{J_{m} J_{l}}\left(\frac{1}{J_{m}}+\frac{1}{J_{l}}\right)\right) \tau_{f} \\
& +\frac{D_{h}}{J_{l}^{2}} \dot{\tau}_{d}+\left(\frac{K_{h}}{J_{l}^{2}}-\frac{D_{h}^{2}}{J_{l}^{2}}\left(\frac{1}{J_{m}}+\frac{1}{J_{l}}\right)\right) \tau_{d} .
\end{aligned}
$$

Note that $u$ is a newly defined control input and $\theta_{l}$ is the output to be controlled. Once $u$ is determined, the actual control input $\tau_{m}$ is obtained by lowpass filtering $u$ using 


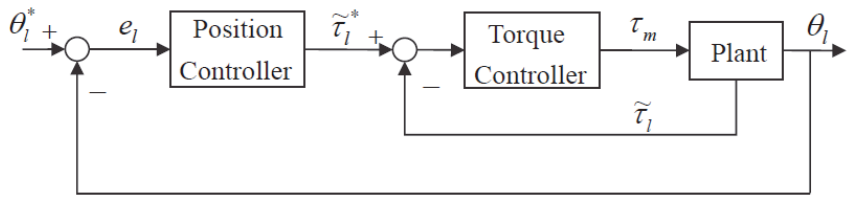

Fig. 2. Schematic of the SSBD for a harmonic drive system.

\subsection{SSBD Design for a Harmonic Drive System}

A harmonic drive system comprises two subsystems. One is described by (10), and the other is given by (11). The SSBD that is an improved CIBD has been proposed ${ }^{(6)}$. For the fourth-order harmonic drive system, the CIBD requires four design steps and it is hard to choose appropriate parameters. On the other hand, the SSBD requires only two design steps for this system that contains two subsystems, as shown in Fig. 2, and it enables the designer to choose proper parameter values for specified transient performance. Let $\theta_{l}^{*}$ denote the reference trajectory that is at least fourth differentiable with respect to time. For the SSBD, define a switching function

$$
\begin{aligned}
& \sigma_{C 0}=\dot{\theta}_{l}^{*}-\dot{\theta}_{l}+C_{1}\left(\theta_{l}^{*}-\theta_{l}\right) \\
& \sigma_{C 1}=\dot{\tilde{\tau}}_{l}^{*}-\dot{\widetilde{\tau}}_{l}+\lambda_{2}\left(\widetilde{\tau}_{l}^{*}-\widetilde{\tau}_{l}\right)+\lambda_{1} \int\left(\widetilde{\tau}_{l}^{*}-\widetilde{\tau}_{l}\right) d t,
\end{aligned}
$$

in which $C_{1}, \lambda_{2}$ and $\lambda_{1}$ are design parameters, and

$$
\widetilde{\tau}_{l}^{*}=-\frac{m g l}{J_{l}} \sin \theta_{l}+\frac{\tau_{d}}{J_{l}}+\ddot{\theta}_{l}^{*}+C_{1}\left(\theta_{l}^{*}-\theta_{l}\right)+C_{0} \sigma_{C 0} .
$$

Here, $C_{0}$ is another design parameter. Following the design procedure demonstrated in the study ${ }^{(6)}$, one has

$$
\begin{aligned}
u= & -f-d_{2}+\ddot{\widetilde{\tau}}_{l}^{*}+\lambda_{2}\left(\dot{\widetilde{\tau}}_{l}^{*}-\dot{\tilde{\tau}}_{l}\right) \\
& +\lambda_{1}\left(\dot{\sigma}_{C 0}+C_{0} \sigma_{C 0}\right)+\lambda_{0} \sigma_{C 1}+\psi_{c} \operatorname{sat}\left(\sigma_{C 1}, \varepsilon\right),
\end{aligned}
$$

in which $\psi_{c}$ is a switching gain, $\varepsilon$ is a positive constant,

$\operatorname{sat}(\cdot, \cdot)$ denotes the saturation function,

$$
\begin{aligned}
& \dot{\tilde{\tau}}_{l}^{*}=-\frac{m g l}{J_{l}} \dot{\theta}_{l} \cos \theta_{l}+\frac{\dot{\tau}_{d}}{J_{l}}+\dddot{\theta}_{l}^{*}+C_{1}\left(\ddot{\theta}_{l}^{*}-\ddot{\theta}_{l}\right)+C_{0} \dot{\sigma}_{C 0}, \\
& \ddot{\widetilde{\tau}}_{l}^{*}=\frac{m g l}{J_{l}} \dot{\theta}_{l}^{2} \sin \theta_{l}-\frac{m g l}{J_{l}} \ddot{\theta}_{l} \cos \theta_{l}+\frac{\ddot{\tau}_{d}}{J_{l}}+\theta_{l}^{*(4)}
\end{aligned}
$$

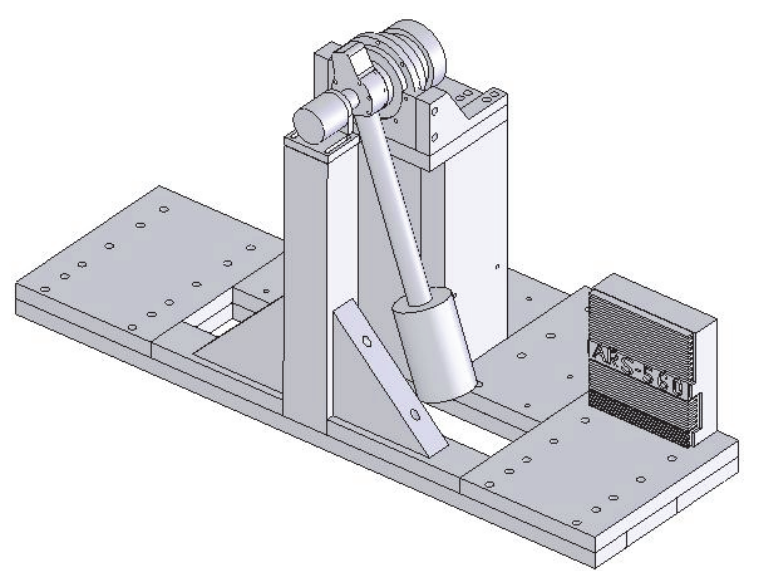

Fig. 3. Schematic of the harmonic drive system.

$$
+C_{1}\left(\dddot{\theta}_{l}^{*}-\dddot{\theta}_{l}\right)+C_{0} \ddot{\sigma}_{C 0}
$$

In practice, $\dddot{\theta}_{l}$ that is required to evaluate $\ddot{\widetilde{\tau}}_{l}^{*}$ is obtained by differentiating $\ddot{\theta}_{l}$ that is measured by an accelerometer.
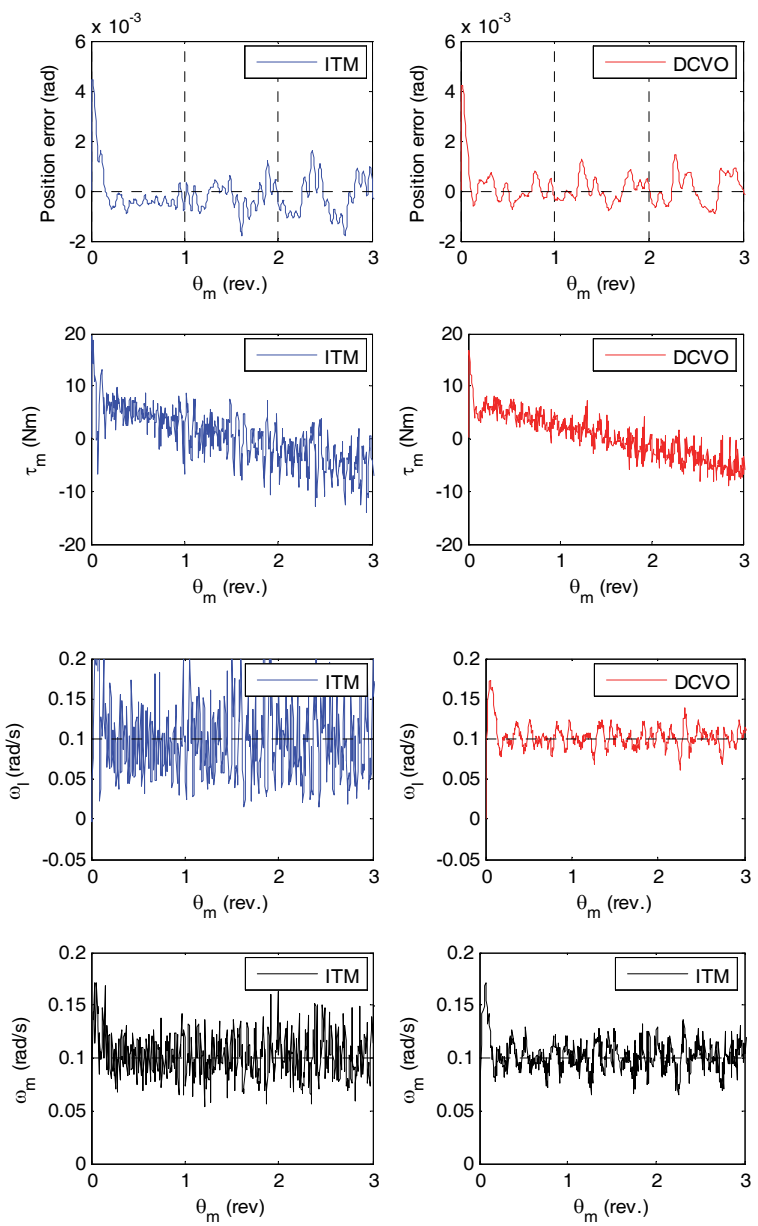

Fig. 4. Tracking control with $\theta_{l}^{*}=0.1 t \quad(\mathrm{rad})$. 

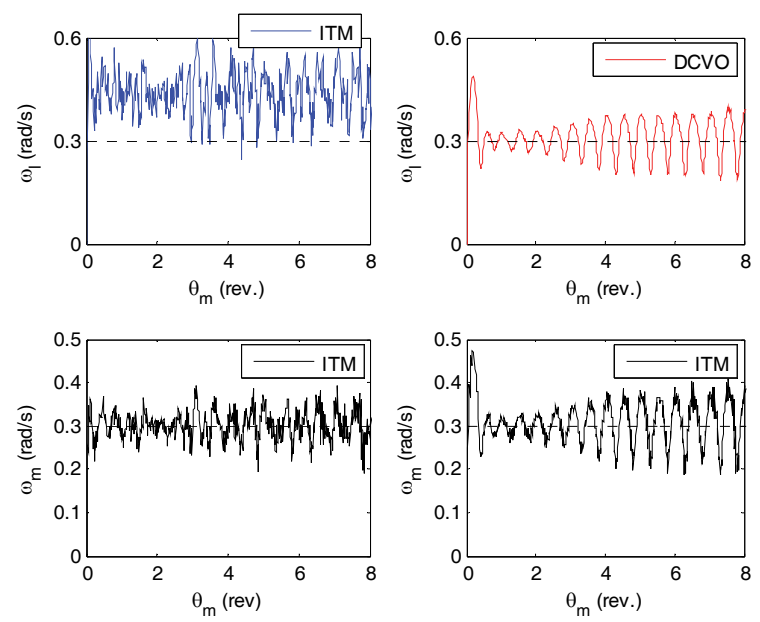

Fig. 5. Tracking control with $\theta_{l}^{*}=0.3 t \quad(\mathrm{rad})$.
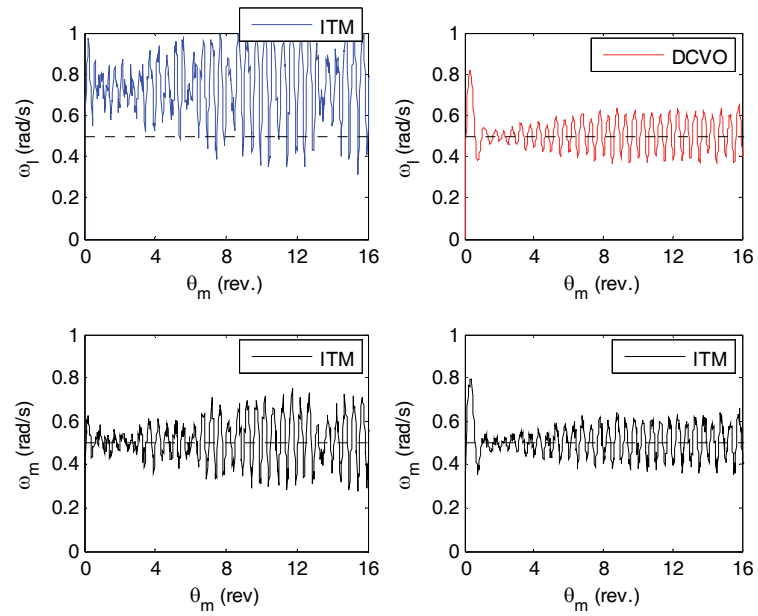

Fig. 6. Tracking control with $\theta_{l}^{*}=0.5 t$ ( $\left.\mathrm{rad}\right)$.
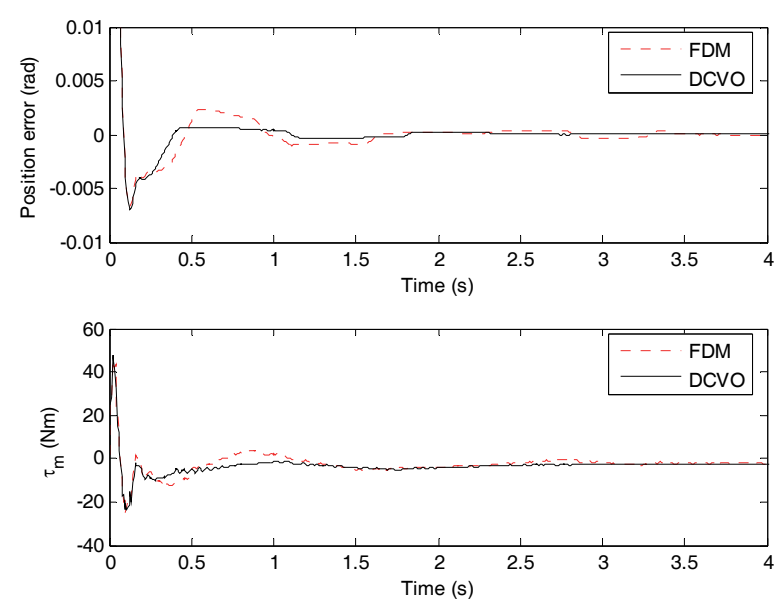

Fig. 7. Tracking control with $\theta_{l}^{*}=0.03$ (rad).

\section{Experimental Harmonic Drive System and Experimental Results}

\subsection{Experimental Harmonic Drive System}

Schematic of the experimental harmonic drive system is shown in Fig. 3, in which the harmonic drive actuator is a hollow-shaft actuator, whose model is SD-25B from Sensodrive $\mathrm{GmbH}$. Please refer to the work $^{(6)}$ for details on the hardware description of this system. The process of system identification was reported in the study ${ }^{(11)}$, giving

$\left[\begin{array}{lllll}\frac{D_{m}}{J_{m}} & D_{h} & \frac{1}{J_{m}} & \frac{1}{J_{l}} & K_{h}\end{array}\right]=\left[\begin{array}{lllll}24 & 10 & 1.8 & 0.8 & 12900\end{array}\right]$

in SI unit. Moreover, $m g l=35.4(\mathrm{Nm})$.

For the SSBD, choose $C_{0}=C_{1}=\omega_{n}, \lambda_{2}=2 \gamma \omega_{n}$, and $\lambda_{1}=\left(\gamma \omega_{n}\right)^{2}$, in which $\gamma=2$ and $\omega_{n}=30$. Moreover, let $\psi_{c}=130000, \varepsilon=2000$ and $\lambda_{0}=\gamma \omega_{n}$ for the SSBD. The sampling frequency of a controller is $12.2 \mathrm{kHz}$, giving $T_{s}=0.082(\mathrm{~ms})$ for the FDM. The ITM is implemented in an FPGA with a counting clock of $100 \mathrm{MHz}$. A load-side encoder of model CP-850-HHC-400K-S-B is used, whose resolution is $1600 \mathrm{k}$ pulses/rev after quadrature decoding. An accelerometer is attached to the payload in order to measure $\ddot{\theta}_{l}$. For the SSVO, choose $L_{1}=2 \omega_{\mathrm{SS}}$ and $L_{2}=\omega_{\mathrm{SS}}^{2}$, in which $\omega_{\mathrm{SS}}=300$. For the DCVO, let

$$
s^{3}+c_{3} s^{2}+c_{2} s+c_{1}=\left(s+6 \omega_{\mathrm{DC}}\right)\left(s^{2}+2 \omega_{\mathrm{DC}} s+\omega_{\mathrm{DC}}^{2}\right),
$$

in which $\omega_{\mathrm{DC}}=83.2$. A motor-side encoder is attached to the motor for measuring $\theta_{m}$. In the following experiments, $\dot{\theta}_{m}$ is estimated using the ITM only, whereas $\dot{\theta}_{l}$ is evaluated using various estimation algorithms for performance comparisons.

\subsection{Comparisons of ITM and DCVO}

Fig. 4 shows tracking responses of the ITM and the DCVO subject to a reference, $\theta_{l}^{*}=0.1 t$ ( $\left.\mathrm{rad}\right)$, in which 
the subplots on the right-hand side show the responses with $\dot{\theta}_{l}$ estimated using the DCVO. It is seen that the DCVO produces a smoother position response than the ITM. Figs. 5 and 6 show responses subject to $\theta_{l}^{*}=0.3 t \quad(\mathrm{rad})$ and $\theta_{l}^{*}=0.5 t \quad(\mathrm{rad})$, respectively, in which $\omega_{\mathrm{m}}$ and $\omega_{1}$ respectively denote $\dot{\theta}_{m}$ and $\dot{\theta}_{l}$. It is seen that there are offsets in the estimate of load-side velocity using the ITM, whereas the DCVO does not produce offsets in estimating the load-side velocity. Note that the ITM modules implemented in an FPGA for both $\omega_{\mathrm{m}}$ and $\omega_{1}$ are the same. The ITM module for $\omega_{\mathrm{m}}$ does not produce offsets in estimating the motor-side velocity. From Figs. 4-6, it is seen that the harmonic drive gearing introduces a disturbance, whose frequency is twice the motor velocity. Moreover, it is implied that the DCVO outperforms the ITM in terms of smoothness and offsets in velocity estimation.

\subsection{Comparisons of FDM and DCVO}

Fig. 7 shows tracking responses of the FDM and the DCVO subject to a reference, $\theta_{l}^{*}=0.03(\mathrm{rad})$, in which the response is the average of responses in five consecutive experiments. It is seen that the DCVO makes the output response less oscillatory than the FDM. The DCVO also improves the positioning performance compared to the FDM.

\subsection{Comparisons of TD and DCVO}

In the experiment that follows, only the velocity estimate by the DCVO is used for feedback control whereas TDs with various values of $\alpha$ are executed simultaneously just to observe velocity for performance comparisons. Figs. 8-10 show results of velocity estimation by the TD with $\alpha=5,3$ and 1, respectively. The upper subplots of Figs. 8-10 are with $\theta_{l}^{*}=0.1 t \quad(\mathrm{rad})$; their middle subplots are with $\theta_{l}^{*}=0.3 t \quad(\mathrm{rad})$; and their lower subplots are with $\theta_{l}^{*}=0.5 t(\mathrm{rad})$. It is seen that the gain of $\alpha=5$ is large enough for $\theta_{l}^{*}=0.5 t \quad$ (rad) but is too large for $\theta_{l}^{*}=0.1 t \quad(\mathrm{rad})$. The gain of $\alpha=3$ is also too large for $\theta_{l}^{*}=0.1 t \quad(\mathrm{rad})$ but becomes insufficient for $\theta_{l}^{*}=0.5 t \quad$ (rad). Reducing the gain to $\alpha=1$ is then insufficient for both $\theta_{l}^{*}=0.3 t \quad(\mathrm{rad})$ and $\theta_{l}^{*}=0.5 t$ (rad). Therefore, it is hard to select an appropriate gain of the TD that is suitable for various operating conditions.

\subsection{Comparisons of SSVO and DCVO}

Fig. 11 shows responses of the SSVO and the DCVO without an accelerometer offset subject to $\theta_{l}^{*}=0.1 t \quad(\mathrm{rad})$. It is seen that both the SSVO and the DCVO produce similar output responses. With an accelerometer offset of $0.5 \mathrm{~V}$, Fig. 12 shows responses of the SSVO and the DCVO subject to the same reference, $\theta_{l}^{*}=0.1 t(\mathrm{rad})$. It is seen that the DCVO is insensitive to the accelerometer offset whereas the DCVO produces an output offset due to the accelerometer offset. Figs. 13 and 14 show responses subject to $\theta_{l}^{*}=0.3 t \quad(\mathrm{rad})$ and $\theta_{l}^{*}=0.5 t \quad(\mathrm{rad})$, respectively, in which both the SSVO and the DCVO are with and without the accelerometer offset. From Figs. 12-14, it is clear that the DCVO is superior to the SSVO in terms of insensitivity to an accelerometer offset.
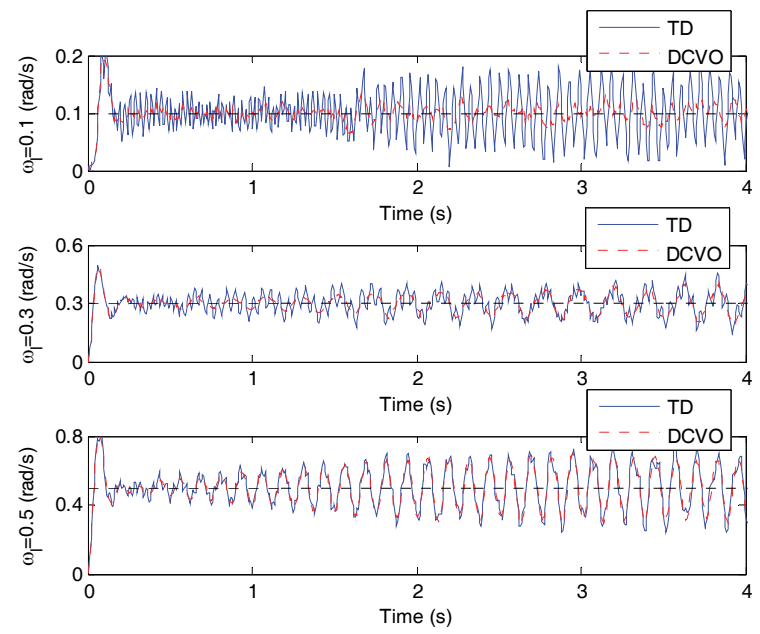

Fig. 8. Velocity estimation by TD with $\alpha=5$, in which feedback control is with the DCVO. 


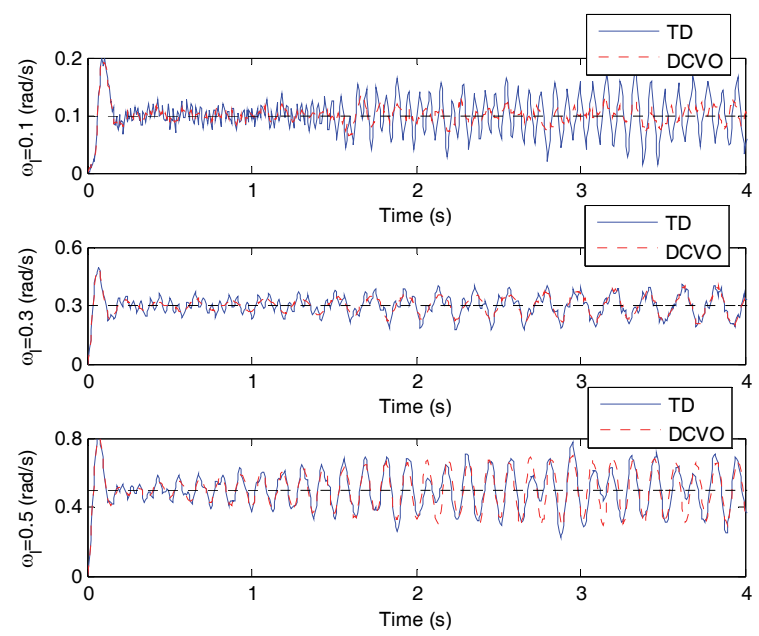

Fig. 9. Velocity estimation by TD with $\alpha=3$, in which feedback control is with the DCVO.
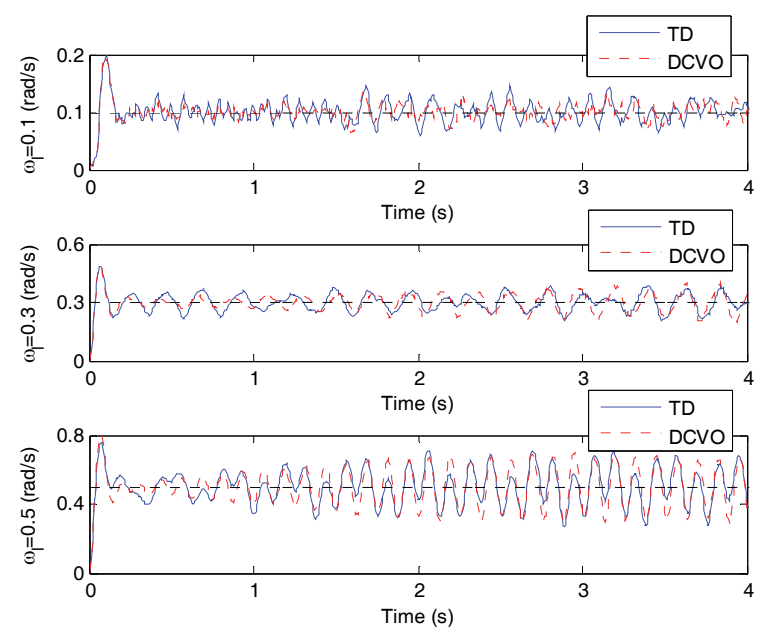

Fig. 10. Velocity estimation by TD with $\alpha=1$, in which feedback control is with the DCVO.
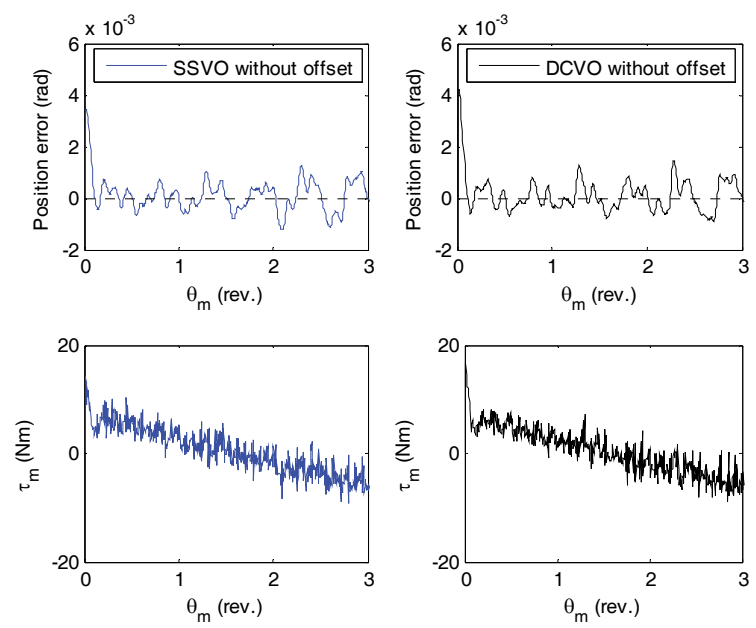

Fig. 11. Responses without an accelerometer offset subject to $\theta_{l}^{*}=0.1 t \quad(\mathrm{rad})$.
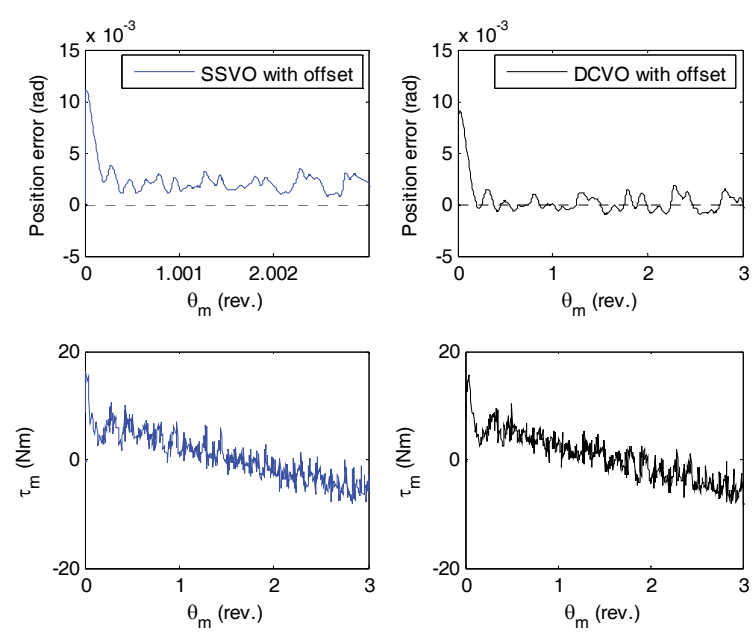

Fig. 12. Responses with an accelerometer offset subject to $\theta_{l}^{*}=0.1 t(\mathrm{rad})$.

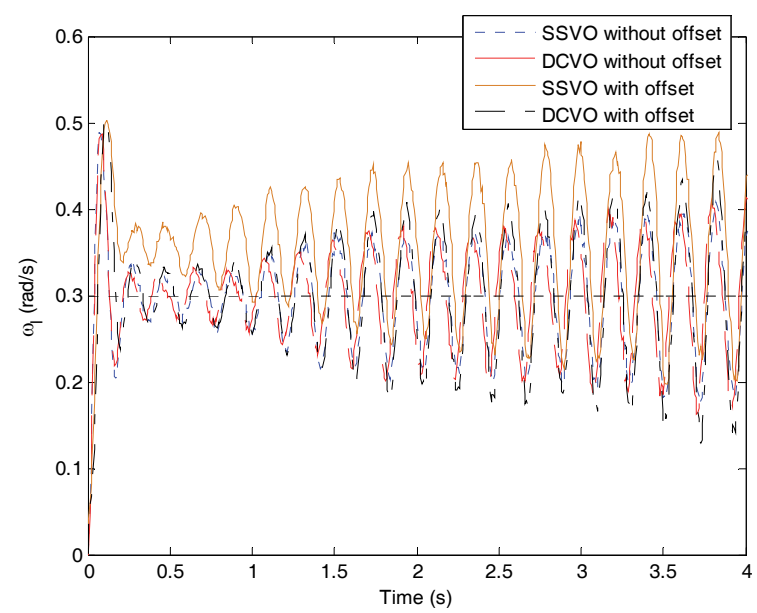

Fig. 13. Responses of SSVO and DCVO subject to $\theta_{l}^{*}=0.3 t(\mathrm{rad})$.

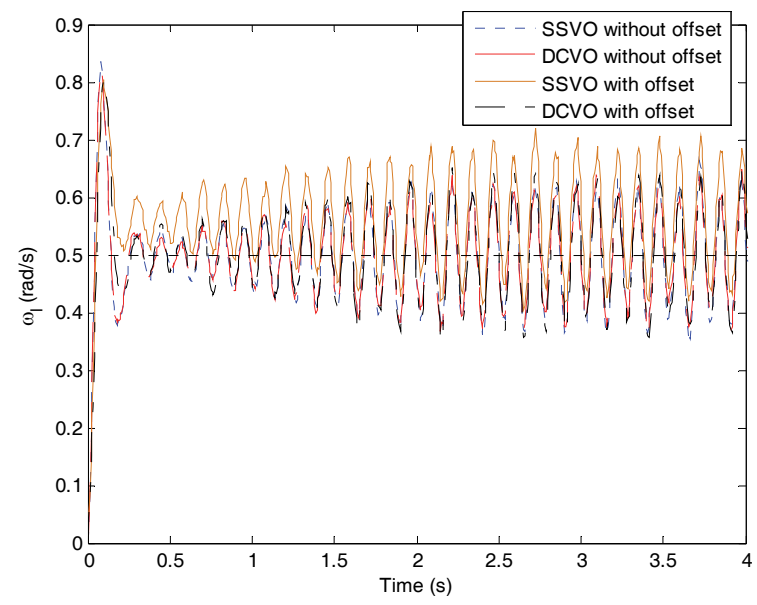

Fig. 14. Responses of SSVO and DCVO subject to $\theta_{l}^{*}=0.5 t(\mathrm{rad})$. 


\section{Conclusions}

Several nonmodel-based velocity estimation schemes have been revisited in this paper, including the ITM, the FDM, the TD, the SSVO and the DCVO. The ITM and the FDM are most commonly used conventional approaches to velocity estimation. However, compared with the DCVO, the ITM produces noisy velocity estimate. The ITM yields poorer tracking performance than the DCVO. For the TD, it is uneasy to choose a proper gain that is suitable for various operating condition. Both the SSVO and the DCVO are assisted by an accelerometer. The SSVO is however sensitive to an accelerometer offset compared to the DCVO. Among these approaches, the DCVO gives best performance in terms of smoothness, tracking performance, and insensitivity to an accelerometer offset.

\section{Acknowledgment}

The authors are grateful to the Ministry of Science and Technology, Taiwan, for supporting this research under Grant MOST 104-2221-E-003-011-MY2. The supports from Prof. G. Hirzinger, N. Sporer, M. Haehnle and M. Hauschild are also gratefully acknowledged. The authors also appreciate advices given by Prof. J.C. Renn with the Department of Mechanical Engineering, National Yunlin Univerity of Science \& Technology, Yunlin, Taiwan.

\section{References}

(1) L. Bascetta, G. Magnani, and P. Rocco : "Velocity estimation: assessing the performance of non-model-based techniques", IEEE Trans. Control Systems Technology, Vol. 17, No. 2, pp. 424-33, 2009

(2) A. Albu-Schaffer, C. Ott, and G. Hirzinger : "A unified passivity-based control framework for position, torque and impedance control of flexible joint robots", Int. J. Robot. Res., Vol. 26, No. 1, pp. 23-39, 2007

(3) W. H. Zhu : "FPGA-based velocity estimation for control of harmonic drives", in Proc. 2010 IEEE Int. Conf. Mechatronics and Automation, Xi'an, 2010, pp. 1069-1074

(4) H. Cai, Y. Huang, and J. Cheng : "Research and application of harmonic drive in mechanical system", in Proc. Int. Conf. Structural, Mechanical and Materials Engineering (ICSMME 2015), Dec. 2015, pp. 161-163

(5) H. Cai, Y. Huang, T. Tang, Q. Wang, J. Du, and T. Zhang : "Double speed loops control for high-precision position tracking of position-controlled actuators involving a harmonic drive reducer", Advances in Mechanical Engineering, Vol. 8, No. 3, pp. 1-10, 2016

(6) Y. S. Lu, C. S. Hwang, and S. F. Lien : "Subsystem backstepping design for controlling a class of nonlinear SISO systems with cascade structure", Mechatronics, Vol. 21, No. 6, pp. 971-982, Sep. 2011

(7) J.-Q. Han : "From PID to active disturbance rejection control", IEEE Trans. Ind. Electron. Vol. 56, No. 3, pp. 900-906, 2009

(8) J. C. Zheng, and M. Y. Fu : "A reset state estimator using an accelerometer for enhancedmotion control with sensor quantization", IEEE Trans. Contr. Syst. Technol., Vol. 18, No. 1, pp. 79-90, 2010

(9) Y. S. Lu, and S. H. Liu : "The design and implementation of an accelerometer-assisted velocity observer”, ISA Trans., Vol. 59, pp. 418-423, Nov. 2015

(10) Y. S. Lu, and C.-H. Lee : "Experimental evaluation of acceleration-enhanced velocity estimation algorithms using a linear motion stage", J. Brazilian Society of Mechanical Sciences and Engineering, Vol. 39, No. 2, pp. 543-551, Feb. 2017

(11) C. S. Hwang, and Y. S. Lu : "Parameter identification and position control of harmonic drive actuators", in Proc. 2005 CACS Automatic Control Conference, Tainan, Taiwan, Nov. 18-19, 2005, pp. d-one-109 d-one-114 